\title{
Early complications after laparoscopic resection of choledochal cyst
}

\author{
Bing Zhang ${ }^{1}$ - Dianming Wu ${ }^{1} \cdot$ Yifan Fang $^{1} \cdot$ Jianxi Bai ${ }^{1}$. Wenhua Huang ${ }^{1} \cdot$ Mingkun Liu $^{1} \cdot$ Jiancai Chen $^{1} \cdot$ Le Li $^{2}$
}

Accepted: 16 May 2019 / Published online: 27 May 2019

(c) The Author(s) 2019

\begin{abstract}
Purpose To investigate the causes and treatments of early complications involving laparoscopic radical resection of choledochal cyst and summarize the experience.

Methods Children with choledochal cyst treated by laparoscopy in the Department of Pediatric Surgery, Fujian Provincial Maternity and Children's Hospital, and Guangzhou Women and Children's Medical Centre, from March 2016 to May 2018, were retrospectively analysed. Demographics, causes and treatments of early complications, liver function analysis and ultrasonography were collected.

Results In total, 231 cases were included; 204 were Type I (156 Type Ia and 46 Type Ic) and 27 were Type IV. No mortality was observed, and 224 cases were successfully laparoscopically operated, while 7 cases were converted to open surgery. Fifteen cases of postoperative developed biliary fistula. There were jejunal Roux loop obstruction in 2 cases and multiple intussusception, anastomotic stenosis after hepaticojejunostomy, residual of choledochal cyst and pancreatic fistula in one each. Patients were followed up ranging from 4 months to 48 months (12.6 \pm 0.3 months on average). Postoperative ALT, AST, GGT, TBIL and DBIL all returned to normal during this time. Ultrasonography indicated 5 cases of widened Glisson's sheath and 1 case of intrahepatic hyperdense shadow.

Conclusion Early complications of laparoscopic radical resection of choledochal cyst can be minimized by properly managing preoperative indications and contraindications, carefully interpreting the magnetic resonance cholangiopancreatography results and accumulating experience by the surgeons.
\end{abstract}

Keywords Choledochal cyst $\cdot$ Laparoscopy $\cdot$ Complications

\section{Introduction}

Choledochal cyst frequently occurs in Asian, mainly in girls, and $80 \%$ of patients have their onset in childhood. Infants and younger children often demonstrate painless jaundice, while older children have recurrent abdominal pain [1]. It is a common disease in departments of paediatric surgery. With the development of imaging techniques and the prenatal screening, an increasing number of asymptomatic cases have been diagnosed [2]. Unless completely resected, the cyst can lead to recurrent symptoms and malignant transformation. Therefore, radical resection of choledochal cysts and

Dianming Wu

879738289@qq.com

1 Department of Pediatric Surgery, Fujian Provincial Maternity and Children's Hospital, Fuzhou 350001, China

2 Department of Pediatric Surgery, Guangzhou Women and Children's Medical Centre, Guangzhou 510623, China
Roux-en-Y hepaticojejunostomy are the ultimate effective treatments [3]. Laparoscopic resection of choledochal cyst has been widely used, but its early complications occurred frequently and should be given more attention. The purpose of this study is to analyse the causes of early complications after laparoscopic resection of choledochal cyst and summarize the experience of treatments.

\section{Materials and methods}

This study presents a retrospective review of paediatric patients with choledochal cyst treated by laparoscopy in the Department of Pediatric Surgery, Fujian Provincial Maternity and Children's Hospital, and Guangzhou Women and Children's Medical Centre, from March 2016 to May 2018. Demographics such as gender, age, weight, clinical manifestation and Todani classification type [4] were collected. The surgery was performed with the four-hole method, as 
the trocars were located at the middle of the umbilicus, right hypochondrium, right side of the abdomen and left hypochondrium, respectively. After dissection of the gallbladder, bile was obtained for bacterial culture and amylase assay, and then, intraoperative cholangiography was performed. An monopolar electrocautery hook or a bipolar coagulation or ultrasonic scalpel was used to dissect the choledochal cyst. In cases of large cyst, the cyst was exposed by suspending the cystic wall using transabdominal sutures. The cyst was dissected down to the distal congenital bile duct dilatation to within the head of the pancreas, and it was then ligated. The upper part of the cyst was further dissected up to the level of the hilar duct and then removed at this level. The umbilical incision was extended to $2-2.5 \mathrm{~cm}$, the jejunum that was 15-20 cm distal from the Treitz ligament was then extracted out of the incision and amputated, and a Roux-en-Y anastomosis was constructed, with a Roux loop length of approximately 15 to $30 \mathrm{~cm}$. A retrocolic end-to-side hepaticojejunostomy was carried out laparoscopically. The mesenteric defects around the Roux loop in the transverse mesocolon and in the small bowel mesentery near the jejuno-jejunostomy routinely closed. A drainage tube was installed at the site of the right upper abdominal trocar. The operation time, blood loss and early complications were collected. Patients were followed up with liver function examinations and colour Doppler ultrasonography.

\section{Results}

A total of 231 cases (42 boys and 189 girls) underwent laparoscopic choledochal cyst excision surgery during the study period. The age ranged from 1 to 139 month with an average of $7.2 \pm 4.3$ months; the weight ranged from 3.2 to $29.4 \mathrm{~kg}$ with an average of $7.3 \pm 2.5 \mathrm{~kg}$. Among them, 197 manifested abdominal pain, 135 had jaundice, 15 had acholic stools, 117 had abdominal masses, 132 had significantly elevated liver function indicators, and 79 had blood/urine amylase levels more than three times higher than normal. The Todani classification is as follows: 204 cases were Type I (156 Type Ia and 46 Type Ic) and 27 cases were Type IV [4].

No intraoperative and postoperative mortalities were observed, and 224 cases of patients were successively performed with laparoscopic operations; the operation time ranged from 2 to $8.3 \mathrm{~h}$ with an average of $3.8 \pm 0.5 \mathrm{~h}$ and blood loss ranged from 20 to $240 \mathrm{ml}$ with an average of $36 \pm 10.2 \mathrm{ml}$; and intraoperative blood transfusion was needed in 13 cases. Seven cases was converted to open operation, of which 4 cases (one with biliary drainage after bile duct perforation, one with recurrent infections of choledochal cyst, one with a distal cyst residual after laparoscopic radical surgery and one with anastomotic stenosis after laparoscopic hepaticojejunostomy) had difficulties in identifying cystic walls or excessive bleeding when dissecting was due to severe local adhesions, oedema and thickening of the cystic walls. Two cases were due to the need for portoenterostomy, and the other one case was due to intraoperative damage to the hepatic artery.

Fifteen cases of postoperative biliary fistula were observed, of which 13 were cured by non-operative treatments and 2 underwent re-operation by open surgery. Between the 2 cases, one case was found to be caused by hepatic duct perforation and was cured after redo hepaticojejunostomy, and the other case was found accessory hepatic duct and was cured after anastomosis, the accessory hepatic duct to the jejunum. There were 2 cases of jejunal Roux loop stenosis and one case each for multiple intussusception, distal residue of choledochal cyst and anastomotic stenosis after hepaticojejunostomy. All of them underwent re-operation by open surgery. One case of pancreatic fistula was cured through conservative treatment (Table 1). The mean postoperative hospital stay ranged from 6 to 90 days with an average of $6.8 \pm 2.3$ days.

Mean follow-up time was $12.6 \pm 0.3$ months; liver function examination and colour Doppler ultrasonography were performed. Preoperative patients with normal alanine aminotransferase (ALT), aspartate aminotransferase (AST) and gamma-glutamyltransferase (GGT), who also performed normal during postoperative. Patients with elevated preoperative levels returned to normal after 3 weeks or 6 weeks or 12 weeks or 16 weeks or 20 weeks after surgery. Total bilirubin (TBIL) and direct bilirubin (DBIL) returned to normal 1-2 weeks after operation. Ultrasonography indicated 5 cases of widened Glisson's sheath and 1 case of intrahepatic hyperdense shadow.

\section{Discussion}

Choledochal cyst, also known as congenital biliary dilatation, includes partial dilatation of extrahepatic bile duct, common bile duct and pancreaticobiliary duct malformations and dilatation of the intrahepatic bile ducts, but it should strictly exclude acquired or secondary dilatations caused by bile duct stones or malignant tumours [5]. Choledochal cysts can lead to obstructive jaundice, liver damage, liver fibrosis and biliary bleeding, and the reflux of pancreatic juice into the biliary tract may cause a high incidence of biliary tract cancer; meanwhile, the entry of bile into the pancreatic duct may cause pancreatitis [6]. Laparoscopic radical surgery can reduce intraoperative bleeding and shorten fasting time and the length of hospitalization stay [7] and is thus currently a safe and effective method for the treatment of choledochal cyst $[8,9]$. Due to its technical difficulties, complications occur inevitably. Analysis of the causes of complications 


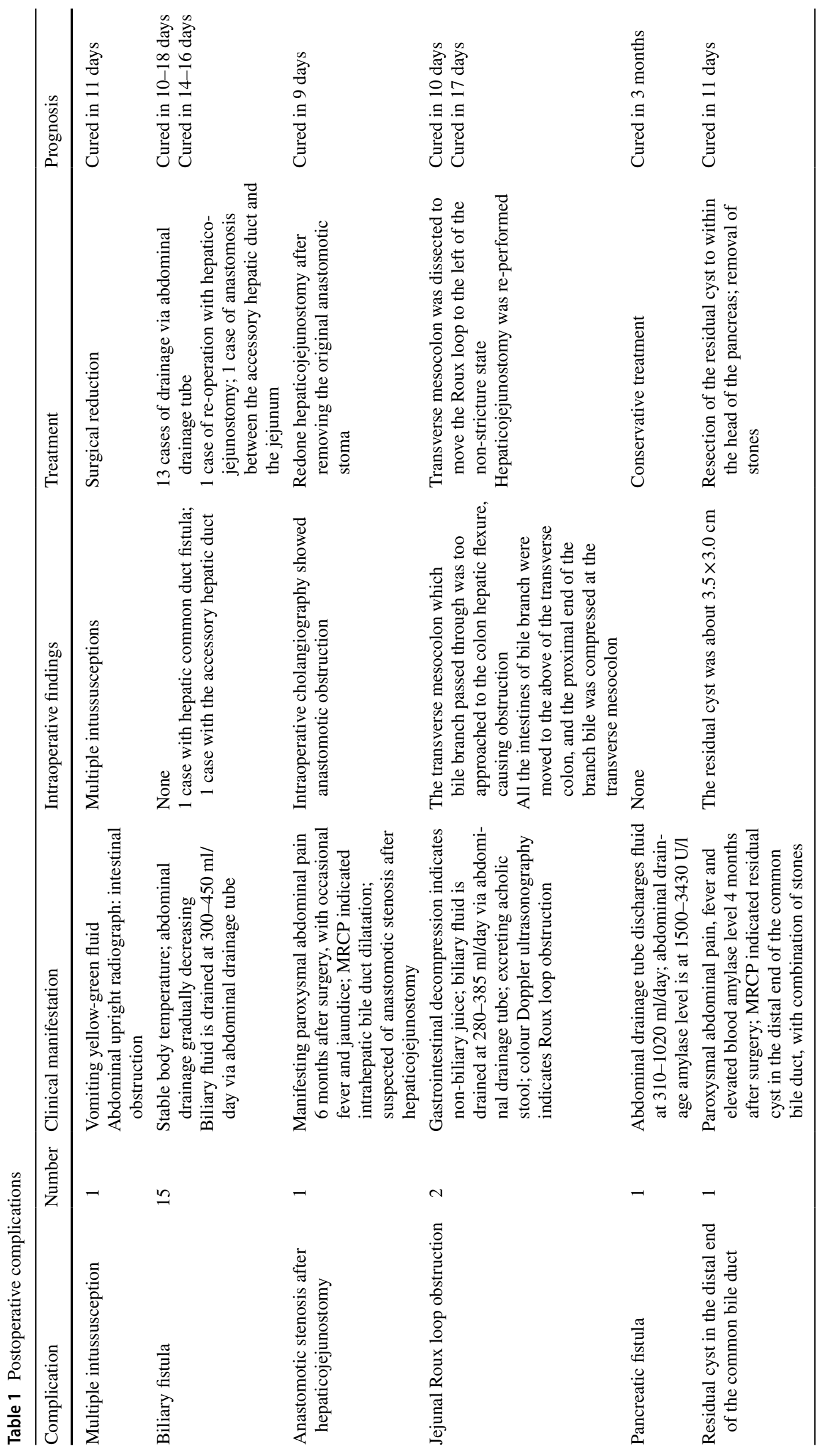


and summarizing the experience of treatments can help to reduce complications and minimize the damage.

Biliary fistula is a common postoperative complication. Its cause is associated with anastomotic tension, anastomosis techniques and blood supply to anastomotic stomata. To prevent this, our experience has been that when dissecting the anterior wall of the cyst, it is recommended to avoid excessively dissecting tissues and completely remove the ulcerous lesions on the inner wall of the cyst. Most cases of biliary fistula can be cured by non-operative treatment. But if the abdominal drainage volume is large or there is no obvious decreasing trend under conservative treatment, it is recommended to re-operate approximately 3 days after initial surgery to avoid intra-abdominal tissue adhesion and oedema aggravation, which are not conducive to recovery after re-surgery. Those two patients with biliary fistula in our study, despite not manifesting fever or abdominal distension, whose drainaged bile were over $300 \mathrm{ml}$ per day, underwent re-operation on the third day after initial surgery.

The incidence of the accessory hepatic duct is $6 \%$ to $20 \%$, and it is another important cause of biliary fistula. It is difficult to be detected by preoperative examination and is easy to be missed intraoperatively, which may result in bile duct infection, cholestasis and localized cirrhosis in the corresponding liver segment [10]. Therefore, we should be highly alert to the accessory hepatic duct when suspicious tubular structure occurs during dissecting the gallbladder or choledochal cyst and further clarification is needed to avoid complications. The accessory hepatic duct observed in this study was not found by preoperative examinations and was spotted as a tubular tissue approximately 2 to $3 \mathrm{~mm}$ in diameter connecting to the cystic duct during dissection of the cystic duct. The accessory hepatic duct was too small to be anastomosed with common hepatic duct. Therefore, it is recommended to ligature the cystic duct near the accessory hepatic duct, incise the residual cystic duct and anastomosis with the lateral wall of the common hepatic duct to form a new anastomosis, which is then anastomosed with the Roux loop (Fig. 1). In one case, a biliary fistula was found to be caused by the accessory hepatic duct. Due to the high discharge of bile drainage, it is not appropriate to perform ligation and instead, we anastomosed the accessory hepatic duct to the jejunum.

Bleeding is also a common complication. The causes mainly include trocar puncture injuries, injury of the gallbladder artery, hepatic artery, or portal vein, bleeding from cystic separation and abnormal coagulation. Our experience has been that in cases of large cysts or severe adhesion, the aspirator and the ultrasonic scalpel are used interchangeably, and coupled with suspending and pulling the cyst with sutures, the posterior wall of the cyst can be gradually dissected. Additional care should be taken on the portal vein and hepatic artery when dissecting the posterior wall of the cyst. It is reported [11] that puncturing the right hepatic artery when suturing the posterior wall during hepaticojejunostomy caused a massive haemorrhage on the third day after surgery, requiring surgical exploration. So it is recommended that when suturing the posterior wall, the anterior wall of the common hepatic duct should be suspended to expose the posterior wall so that vascular damage can be avoided. In cases of active postoperative bleeding after transfusion and correction of coagulopathy, re-operation should be conducted in time.

Biliary obstruction after bile duct reconstruction is not rare. The causes include aberrant right hepatic artery, combination with hepatic duct stenosis, anastomotic stenosis and jejunal Roux loop obstruction. It is recommended to re-operate as soon as possible to reduce liver damage. (1) Undetected right hepatic artery in front of the extrahepatic biliary tract is among the main causes of recurrent biliary obstruction after radical surgery. In biliary tract reconstruction, placing the hepatic artery behind the common hepatic duct (Fig. 2) to restore normal anatomy can avoid this complication [12,13]. (2) Regarding common hepatic duct stenosis, analysis of preoperative imaging and carefully exploring stenosis of the common hepatic duct and the left and right hepatic ducts intraoperatively, the stenotic segment should be opened to normal level. Two patients aged approximately 2 months were found to have the common hepatic duct which was small to approximately $1 \mathrm{~mm}$ in diameter when dissecting the proximal end of the cyst. Although preoperative magnetic resonance cholangiopancreatography (MRCP) did not indicate proximal biliary stenosis or dilatation, intraoperative cholangiography showed that contrast did not enter the left or right hepatic ducts, so we suspected the concurrent common hepatic duct stenosis and opened the duct in the anterior wall. We found that the common hepatic duct was very thin and it is difficult to clearly identify the left and right hepatic ducts even under a magnified laparoscopic field of view; thus, we performed hepatic portal-jejunal anastomosis ultimately (Fig. 3). In this regard, our experience is that if the common hepatic duct is found small, even if the left and right hepatic ducts do not develop on intraoperative cholangiography, as long as the preoperative MRCP does not suggest proximal hepatic duct dilatation and the drainage volume of bile at the opening of common hepatic duct is found to be large during surgery, then we would not consider the combination of stenosis and would not recommend opening the anterior wall of the main hepatic duct. (3) The incidence of stenosis of choledochaljejunal anastomosis after laparoscopic choledochal cyst excision was $1.6 \%$. The main causes include small anastomosis, excessive tension, insufficient blood supply and inflammation or infection associated with biliary leakage $[11,14,15]$. In this study, anastomotic stenosis occurred in one case with biliary fistula 6 months after initial surgery, so the original 

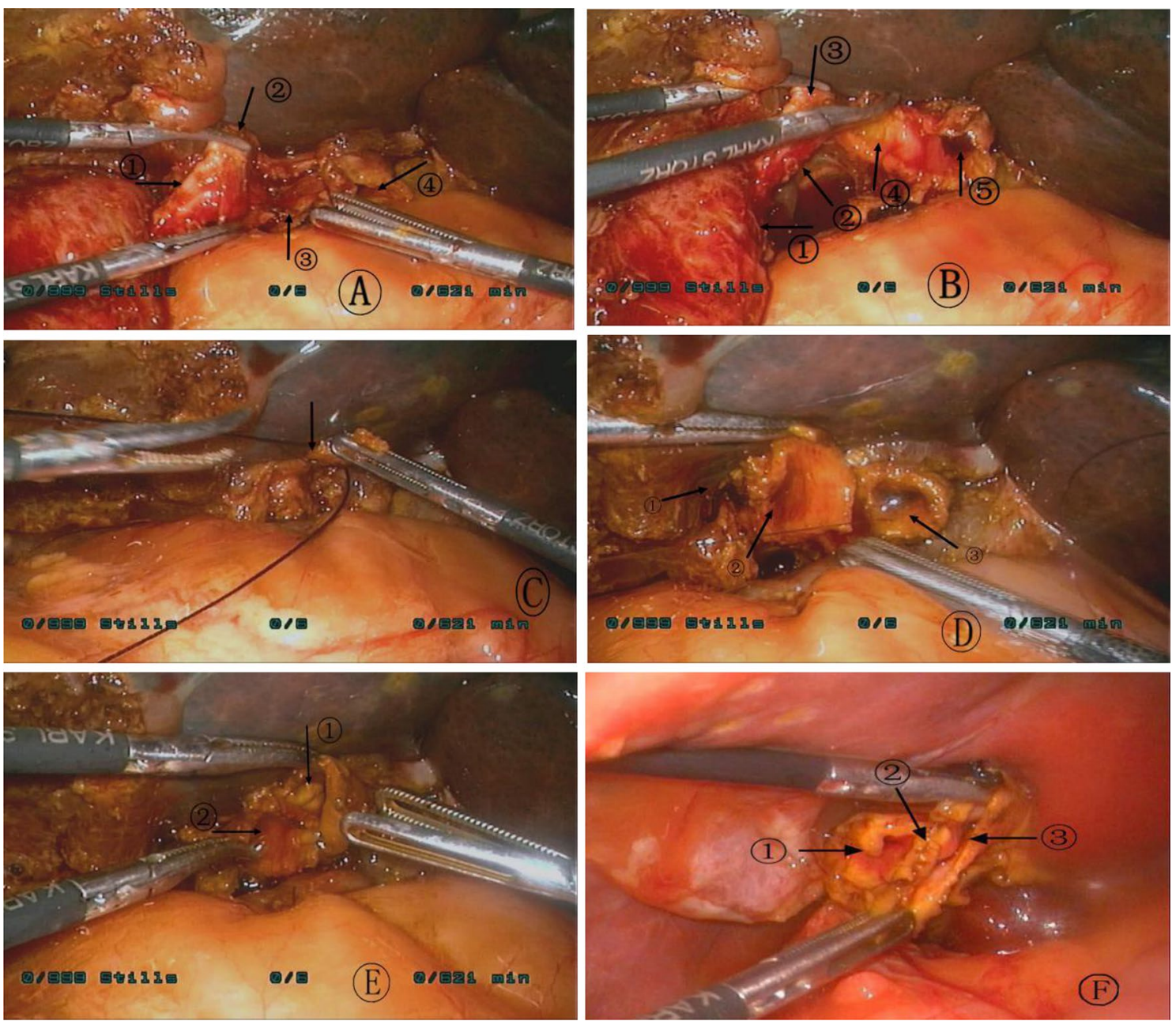

Fig. 1 a Indicated by the arrow: (1) distal end of the cystic duct; (2) accessory hepatic duct; (3) proximal end of the cystic duct; (4) common hepatic duct. b Indicated by the arrow: (1) gallbladder; (2) distal end of the cystic duct; (3) accessory hepatic duct; (4) opened proximal end of the cystic duct; (5) common hepatic duct. $\mathbf{c}$ The diameter of the accessory hepatic duct is approximately $3 \mathrm{~mm}$, making it difficult to anastomose, so the distal end of cystic duct is ligated at the location near the accessory hepatic duct (indicated by the arrow). d Indicated by the arrow: (1) the ligated distal end of the cystic duct (near the acces-

anastomotic stoma was amputated and a new anastomosis was performed; the patient was eventually cured (Fig. 4). So we recommended that measures should be undertaken to ensure no tension on the stoma, good blood supply to the stoma, a sufficiently large stoma diameter (infant $\geqq 1 \mathrm{~cm}$, and newborn $\geq 0.5 \mathrm{~cm}$ ) and that the technique of anastomosis should be improved to avoid stenosis. (4) The incidence of jejunal Roux loop obstruction is not high, mostly related to the twisting caused by excessively long Roux loops [16]. However, the two cases observed in this study occurred in the region at which the Roux loop crosses the transverse mesocolon. They manifested paroxysmal abdominal pain on the first day after radical surgery, and abdominal drainage sory hepatic duct); (2) the trimmed proximal end of the cystic duct; (3) common hepatic duct. e After the anastomosis between the accessory hepatic duct and the common hepatic duct (anastomosis between the trimmed cystic duct and common hepatic duct): (1) common hepatic duct; (2) the trimmed proximal end of cystic duct. f Another patient, after the anastomosis between the accessory hepatic duct and the common hepatic duct: (1) accessory hepatic duct; (2) suture surface; (3) common hepatic duct

tube drained biliary fluid. After non-operative treatment, the abdominal pain was not relieved, ultrasonography indicated jejunal Roux loop dilation, and the patients were re-operated on the third day after surgery. Our experience is that it is necessary to determine the location of the common hepatic duct, so the transverse mesocolon region is identified to ensure the shortest and smoothest path of the Roux loop. Then, the dissection is performed in the avascular region of the transverse mesocolon region with an ultrasonic scalpel, and after passing across the transverse mesocolon region, the Roux loop was lifted again to ensure that no twisting or angulation was present. The seromuscular layer of the mesocolon passing across the transverse mesocolon region 


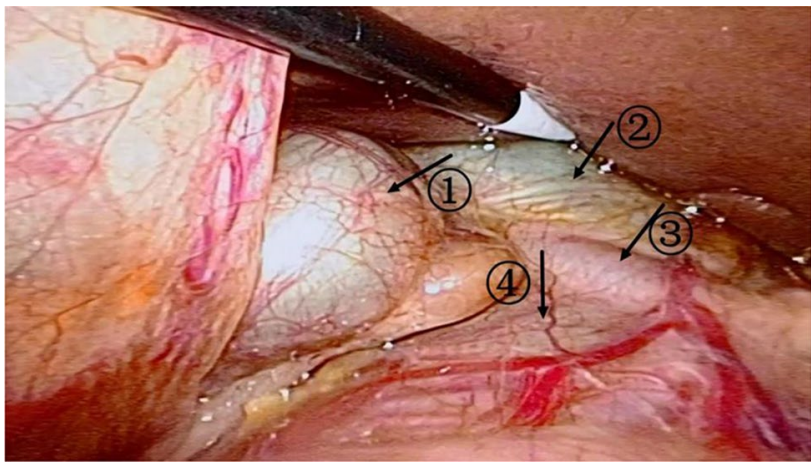

Fig. 2 Indicated by the arrow: (1) cystic duct; (2) proximal end of the common hepatic duct; (3) ectopically presented right hepatic artery; (4) choledochal cyst

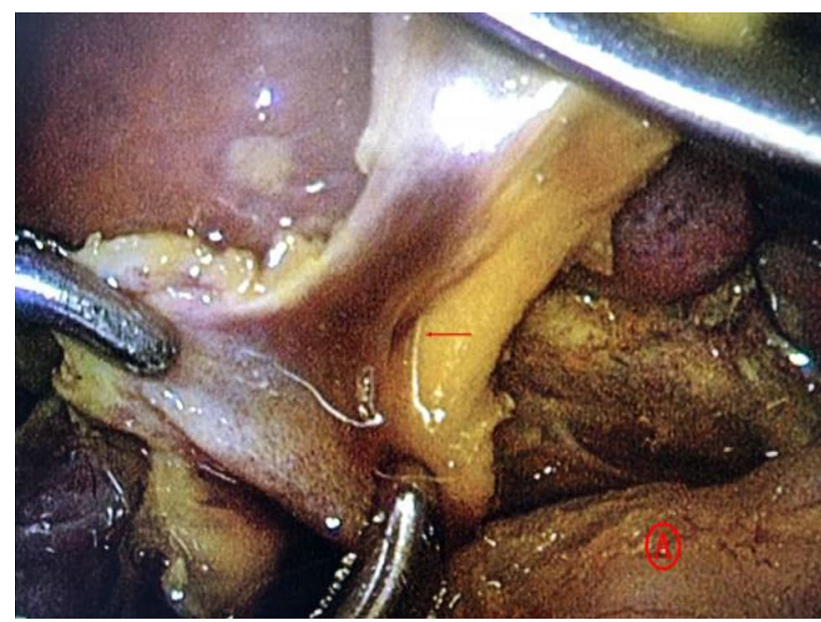

Fig. 3 A. Indicated by the arrow is the fine opening of the common hepatic duct

and the transverse mesocolon were fixed with sutures. After radical surgery, if the patient manifests paroxysmal abdominal pain, a large volume of bile drainage through the abdominal drainage tube, no biliary outflow from gastrointestinal decompression, and the excretion of acholic stools, we should be highly alert to the Roux loop obstruction.

Pancreatic fistula occurs mainly because the distal end of the common bile duct cyst is not close enough to the common wall of the cyst wall. The diagnostic criterion of the International Pancreatic Research Group for pancreatic fistula is that $>3$ days after surgery, the amylase concentration in any amount of drainage fluid is more than three times higher than the upper limit of a normal serum amylase concentration, which is related to the prognosis. An abdominal fluid amylase level of over $2000 \mathrm{U} / \mathrm{l}$ on the first postoperative day may help to diagnose pancreatic fistula $[17,18]$. If pancreatic fistula is suspected after surgery, an additional fine plastic tube can be inserted through the abdominal drainage tube, through which normal saline is continuously perfused and then drained via a thick drainage tube. If the flushing is effective, conservative treatment can be considered; if after the flushing, the abdominal symptoms are aggravated, the body temperature continues to rise. In such a case, flushing through ultrasound-guided intubation or re-operation should be performed.

Postoperative pancreatitis is rare and associated with abnormal pancreatic ducts, such as residual cyst in pancreas [19]. At the early stage in this study, there was a case in which the distal end of the cyst was not completely dissipated by laparoscopic operation to avoid the injury of pancreatobiliary common duct and thus would be in the need for re-operation. In the later cases, the MRCP was read carefully before surgery, and the intraoperative cholangiography was used to identify the pancreatic duct. Pancreatic duct injury could be avoided when dissecting the cyst to the distal. And then suturing after full washing, no similar complications appear (Fig. 5).

Although this study is a two-centre, large-sample study, there are still some limitations: the sample is a continuous sample and there is selective bias. Follow-up time is too short; long-term complications such as anastomotic stricture, biliary calculi, reflux cholangitis and so on need to be further followed up.

\section{Conclusion}

In summary, laparoscopic radical resection of the choledochal cyst has become a mature technique; despite high surgical difficulty and various complications, the proper preoperative management of indications and contraindications and careful interpretation of the MRCP results with the surgeon's accumulation of surgical experience can minimize surgical complications. 

the arrows indicate anastomotic stenosis after hepaticojejunostomy, the significantly dilated proximal end of the common hepatic duct, and the left and right hepatic ducts. b Intraoperative contrast injection into the jejunal Roux loop, indicating: (1) anastomotic stenosis after hepaticojejunostomy; (2) the injection of contrast agent after the distal end of the duct of the Roux loop was blocked with an intestinal clamp
Fig. 4 a MRCP examination;
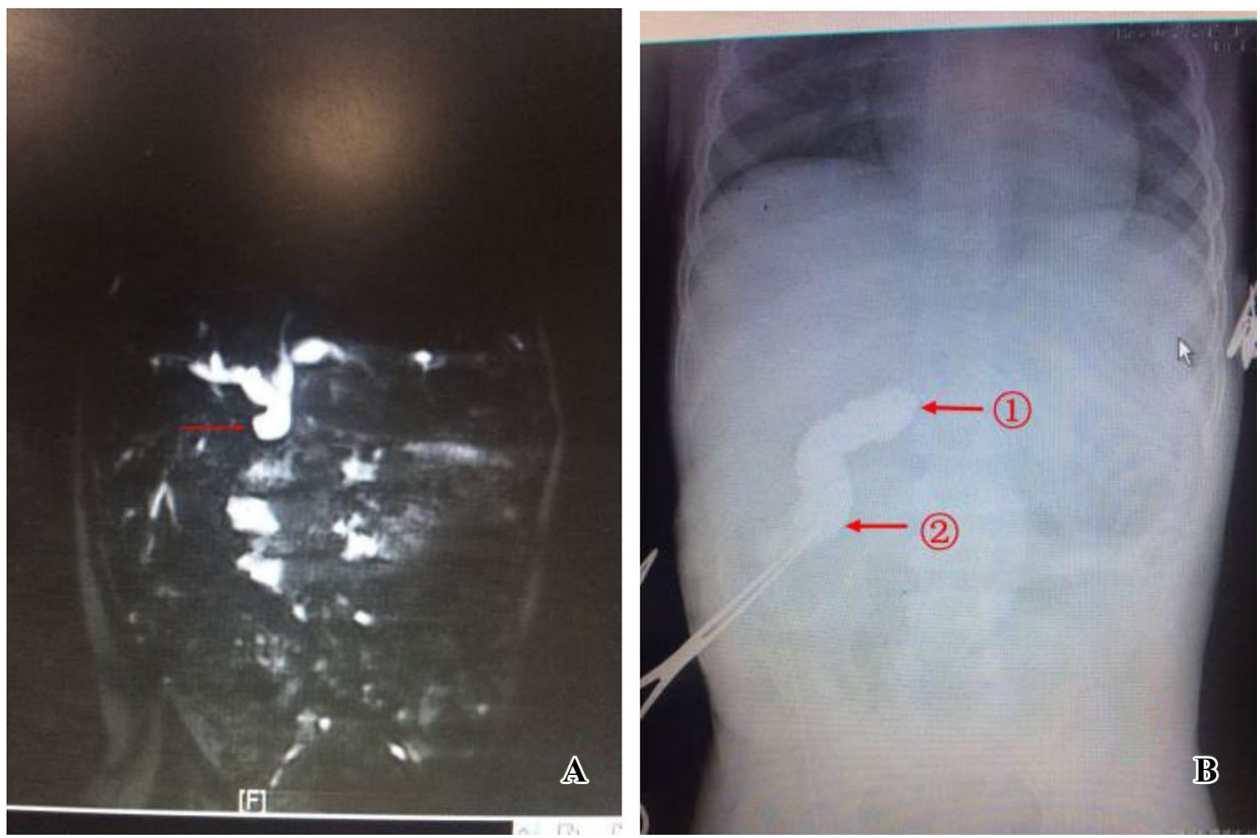

Fig. 5 a Intraoperative cholecystography; the arrow indicates the pancreatic duct. $\mathbf{b}$ Preoperative MRCP examination; the arrow indicates the pancreatic duct
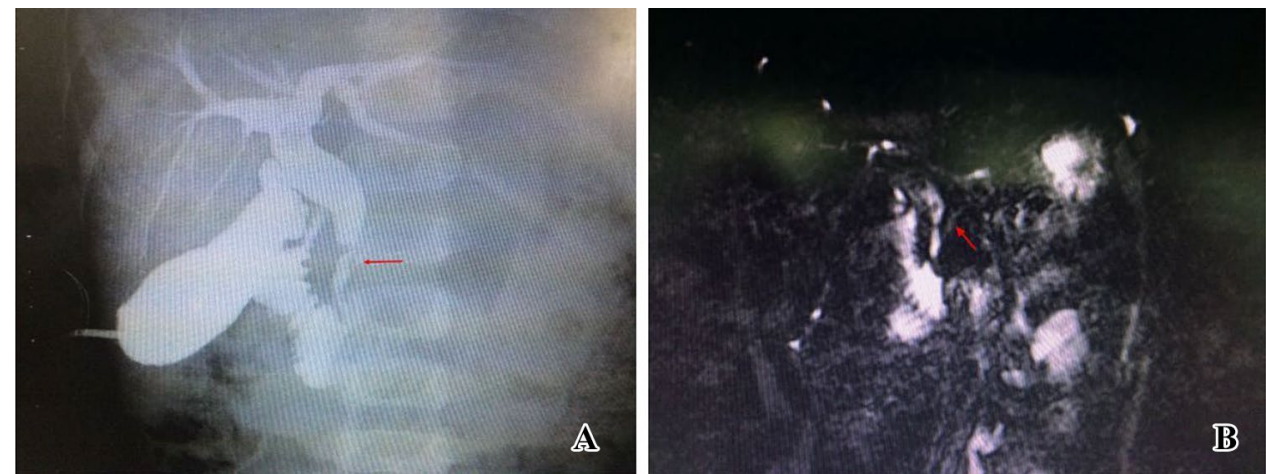

Authors' contributions BZ, the first author, contributed to brainstorming and the design of the experiment, the implementation of the experiments, the collection and analysis of the data, and the drafting of the manuscript; DW, corresponding author, contributed to brainstorming and the design of the experiment, the implementation of the experiment, and the drafting of the manuscript; YF, the second author, contributed to brainstorming and the design of the experiment and the critical reviewing of the specialty knowledge related to the manuscript; $\mathrm{JB}, \mathrm{WH}, \mathrm{ML}, \mathrm{JC}$, the third authors, contributed to brainstorming and the design of the experiment and guided the manuscript revision; LL, the fourth author, contributed to the collection of the data and provided technical support.

\section{Compliance with ethical standards}

Conflict of interests The authors declare that they have no conflicts of interests.

Ethical approval All procedures performed in studies involving human participants were in accordance with the ethical standards of the institutional and/or national research committee and with the 1964 Helsinki Declaration and its later amendments or comparable ethical standards.
Informed consent Informed consent was obtained from all individual participants included in the study.

Open Access This article is distributed under the terms of the Creative Commons Attribution 4.0 International License (http://creativeco mmons.org/licenses/by/4.0/), which permits unrestricted use, distribution, and reproduction in any medium, provided you give appropriate credit to the original author(s) and the source, provide a link to the Creative Commons license, and indicate if changes were made.

\section{References}

1. Makin E, Davenport M (2012) Understanding choledochal malformation. Arch Dis Child 97(1):69-72. https://doi.org/10.1136/ adc. 2010.195974

2. Urushihara N, Fukumoto K, Yamoto M et al (2018) Characteristics, management, and outcomes of congenital biliary dilatation in neonates and early infants: a 20-year, single-institution study. J Hepato-Bil-Pan Sci. https://doi.org/10.1002/jhbp.590 
3. Ronnekleiv-Kelly SM, Soares KC, Ejaz A et al (2016) Management of choledochal cysts. Curr Opin Gastroen 32(3):225. https ://doi.org/10.1097/MOG.0000000000000256

4. Todani T (1997) Congenital choledochal dilatation: classification, clinical features, and long-term results. J Hepato-biliarypan 4(3):276-282. https://doi.org/10.1007/bf02489025

5. Hamada Y, Ando H, Kamisawa T et al (2016) Diagnostic criteria for congenital biliary dilatation 2015. J Hepato-bil-pan Sci 23(6):342-346. https://doi.org/10.1002/jhbp.346

6. Kamisawa T, Ando H, Hamada Y et al (2014) Diagnostic criteria for pancreaticobiliary maljunction 2013. J Hepato-bil-pan Sci 21(3):159-161. https://doi.org/10.1002/jhbp.57

7. Liu Y, Yao X, Li S et al (2014) Comparison of therapeutic effects of laparoscopic and open operation for congenital choledochal cysts in adults. Gastroent Res Pract 3:670260. https:// doi.org/10.1155/2014/670260

8. Qiao G, Li L, Li S et al (2015) Laparoscopic cyst excision and Roux-Y hepaticojejunostomy for children with choledochal cysts in China: a multicenter study. Surg Endosc 29(1):140-144. https://doi.org/10.1007/s00464-014-3667-7

9. Ahmed B, Sharma P, Leaphart CL (2017) Laparoscopic resection of choledochal cyst with Roux-en-Y hepaticojejunostomy: a case report and review of the literature. Surg Endosc 31(8):1-6. https://doi.org/10.1007/s00464-016-5346-3

10. Shaikh IA, Thomas H, Joga K et al (2010) Post-cholecystectomy cystic duct stump leak: a preventable morbidity. J Digest Dis 10(3):207-212. https://doi.org/10.1111/j.1751-2980.2009.00387.x

11. Diao M, Li L, Cheng W (2016) Laparoscopic redo hepaticojejunostomy for children with choledochal cysts. Surg Endosc 30(12):1-7. https://doi.org/10.1007/s00464-016-4915-9

12. Agur AMR, Dalley IIAF (2012) Abdomen-Bliliatry ducts. In: Agur AMR (ed) Grant's atlas of anatomy, 13th edn. Lippincoot Williams \& Wilkins, Philadelphia, pp 160-164
13. Mei D, Long L, Wei C (2016) Recurrence of biliary tract obstructions after primary laparoscopic hepaticojejunostomy in children with choledochal cysts. Surg Endosc 30(9):3910-3915. https:// doi.org/10.1007/s00464-015-4697-5

14. Tang ST, Yang Y, Wang Y et al (2011) Laparoscopic choledochal cyst excision, hepaticojejunostomy, and extracorporeal Roux-en-Y anastomosis: a technical skill and intermediate-term report in 62 cases. Surg Endosc 25(2):416-422. https://doi.org/10.1007/s0046 4-010-1183-y

15. Diao M, Li L (2013) Role of laparoscopy in treatment of choledochal cysts in children. Pediatr Surg Int 29(4):317-326. https:// doi.org/10.1007/s00383-013-3266-Z

16. Liuming H, Hongwu Z, Gang L et al (2011) The effect of laparoscopic excision vs open excision in children with choledochal cyst: a midterm follow-up study. J Pediatr Surg 46(4):662-665. https://doi.org/10.1016/j.jpedsurg.2010.10.012

17. Pulvirenti A, Ramera M, Bassi C (2017) Modifications in the International Study Group for Pancreatic Surgery (ISGPS) definition of postoperative pancreatic fistula. Transl Gastroenterol Hepatol 2(12):107. https://doi.org/10.21037/tgh.2017.11.14

18. Cheng D, Liu N, Zhaodong W et al (2016) Value of drain fluid amylase level obtained on the first postoperative day in prediction of pancreatic fistula. Chin J Gen Surg 25(9):1307-1312. https:// doi.org/10.1002/hed.10272

19. Urushihara N, Fukumoto K, Fukuzawa H et al (2012) Longterm outcomes after excision of choledochal cysts in a single institution: operative procedures and late complications. J Pediatr Surg 47(12):2169-2174. https://doi.org/10.1016/j.jpeds urg.2012.09.001

Publisher's Note Springer Nature remains neutral with regard to jurisdictional claims in published maps and institutional affiliations. 